\title{
Concordance and predictive value of two adverse drug event data sets
}

Aurel Cami ${ }^{1,2^{*}}$ and Ben Y Reis ${ }^{1,2}$

\begin{abstract}
Background: Accurate prediction of adverse drug events (ADEs) is an important means of controlling and reducing drug-related morbidity and mortality. Since no single "gold standard" ADE data set exists, a range of different drug safety data sets are currently used for developing ADE prediction models. There is a critical need to assess the degree of concordance between these various ADE data sets and to validate ADE prediction models against multiple reference standards.
\end{abstract}

Methods: We systematically evaluated the concordance of two widely used ADE data sets - Lexi-comp from 2010 and SIDER from 2012. The strength of the association between ADE (drug) counts in Lexi-comp and SIDER was assessed using Spearman rank correlation, while the differences between the two data sets were characterized in terms of drug categories, ADE categories and ADE frequencies. We also performed a comparative validation of the Predictive Pharmacosafety Networks (PPN) model using both ADE data sets. The predictive power of PPN using each of the two validation sets was assessed using the area under Receiver Operating Characteristic curve (AUROC).

Results: The correlations between the counts of ADEs and drugs in the two data sets were 0.84 (95\% Cl: 0.82-0.86) and 0.92 (95\% Cl: 0.91-0.93), respectively. Relative to an earlier snapshot of Lexi-comp from 2005, Lexi-comp 2010 and SIDER 2012 introduced a mean of 1,973 and 4,810 new drug-ADE associations per year, respectively. The difference between these two data sets was most pronounced for Nervous System and Anti-infective drugs, Gastrointestinal and Nervous System ADEs, and postmarketing ADEs. A minor difference of $1.1 \%$ was found in the AUROC of PPN when SIDER 2012 was used for validation instead of Lexi-comp 2010.

Conclusions: In conclusion, the ADE and drug counts in Lexi-comp and SIDER data sets were highly correlated and the choice of validation set did not greatly affect the overall prediction performance of PPN. Our results also suggest that it is important to be aware of the differences that exist among ADE data sets, especially in modeling applications focused on specific drug and ADE categories.

Keywords: Adverse drug events, Prediction, Concordance

\section{Background}

Predictive modeling of adverse drug events (ADEs) is attracting growing interest. The high ADE-related costs in the US have been known for many years [1,2], and recent studies conducted in other developed countries provide further motivation for the importance of this problem worldwide [3-5]. To address these large and growing ADE-related economic and public health concerns, a wide array of ADE identification and prevention methods have been implemented. These include early-

\footnotetext{
* Correspondence: aurel.cami@childrens.harvard.edu

'Division of Emergency Medicine, Boston Children's Hospital, 1 Autumn Street, 5th Floor, Boston, MA 02215, USA

${ }^{2}$ Department of Pediatrics, Harvard Medical School, Boston, MA, USA
}

stage drug toxicity prediction and testing [6-8], clinical trials for evaluating a drug's safety profile, and postmarket surveillance methods for detecting abnormally high ADE rates $[9,10]$. Still, many types of ADEs can go undetected for years after a drug has been on the market $[11,12]$, necessitating constant additions of label warnings and, in extreme cases, drug withdrawals $[13,14]$. At the same time, toxicity and clinical safety concerns remain lead causes of the high attrition rates in the drug development process [15], as the cost of bringing New Molecular Entities (NMEs) to the market continues to increase [16]. All these factors have spurred a noticeable expansion in research on adverse event prediction - 
research that critically relies on good data. The emerging field of system pharmacology is being increasingly recognized as a promising new approach for predicting ADEs [17]. System pharmacological approaches typically rely on the integration of various diverse types of data, such as chemical, biological and taxonomic, followed by the application of quantitative models to extract information from these data. Often times, the data are represented and integrated through network models $[18,19]$. In recent years, a number of system pharmacology predictive models for ADEs have been proposed [20-27].

While all these predictive approaches rely critically on "known" drug-ADE associations, there is currently no "gold standard" source for drug safety data. As a result, several different ADE data sets have been used to develop predictive pharmacological models. Often, the drug$\mathrm{ADE}$ associations listed in these data sets are primarily extracted from drug package inserts. This is the case, for instance, with SIDER - a widely used public database, and Lexi-comp - a widely used commercial database. Alternatively, the listed drug-ADE pairs may be extracted from post-marketing databases, such as FAERS (formerly AERS; http://www.fda.gov/Drugs). Thus, the drug safety data used to train the above models may contain drug-ADE associations supported by strong evidence (e.g. associations for whom a causal link between the drug and $\mathrm{ADE}$ has rigorously been established) as well as associations supported by weaker evidence (e.g. associations based solely on post-marketing reports).
Recent work has highlighted various types of inconsistencies in the reporting of drug safety information, including discrepancies among the reports for bioequivalent drugs, or reports used in different countries [28-30]. However, systematic comparisons of the major safety data sets used in system pharmacological models and assessment of the impact of data-set choice on prediction performance are lacking. In this paper, we systematically compare the Lexi-comp and SIDER ADE data sets. While the choice of an ADE data set can also have diverse clinical and economic consequences, we focus on its implications for predictive models. As a case study, we use the Predictive Pharmacosafety Networks (PPNs) model.

\section{Methods}

\section{Framework overview}

Figure 1 shows an overview of the study framework. First, we integrated data from multiple sources, including data on drug-ADE associations from two snapshots of Lexi-comp and one snapshot of SIDER, drug and ADE taxonomies, and intrinsic drug properties. Next, we carried out a number of steps to standardize and integrate these data, including mapping the Lexi-comp and SIDER ADE names to MedDRA High Level Terms (HLTs), standardizing the drug names in Lexi-comp and SIDER, and constructing bi-partite network representations of the drug-ADE associations. Next, we assessed the strength of the association between the counts of

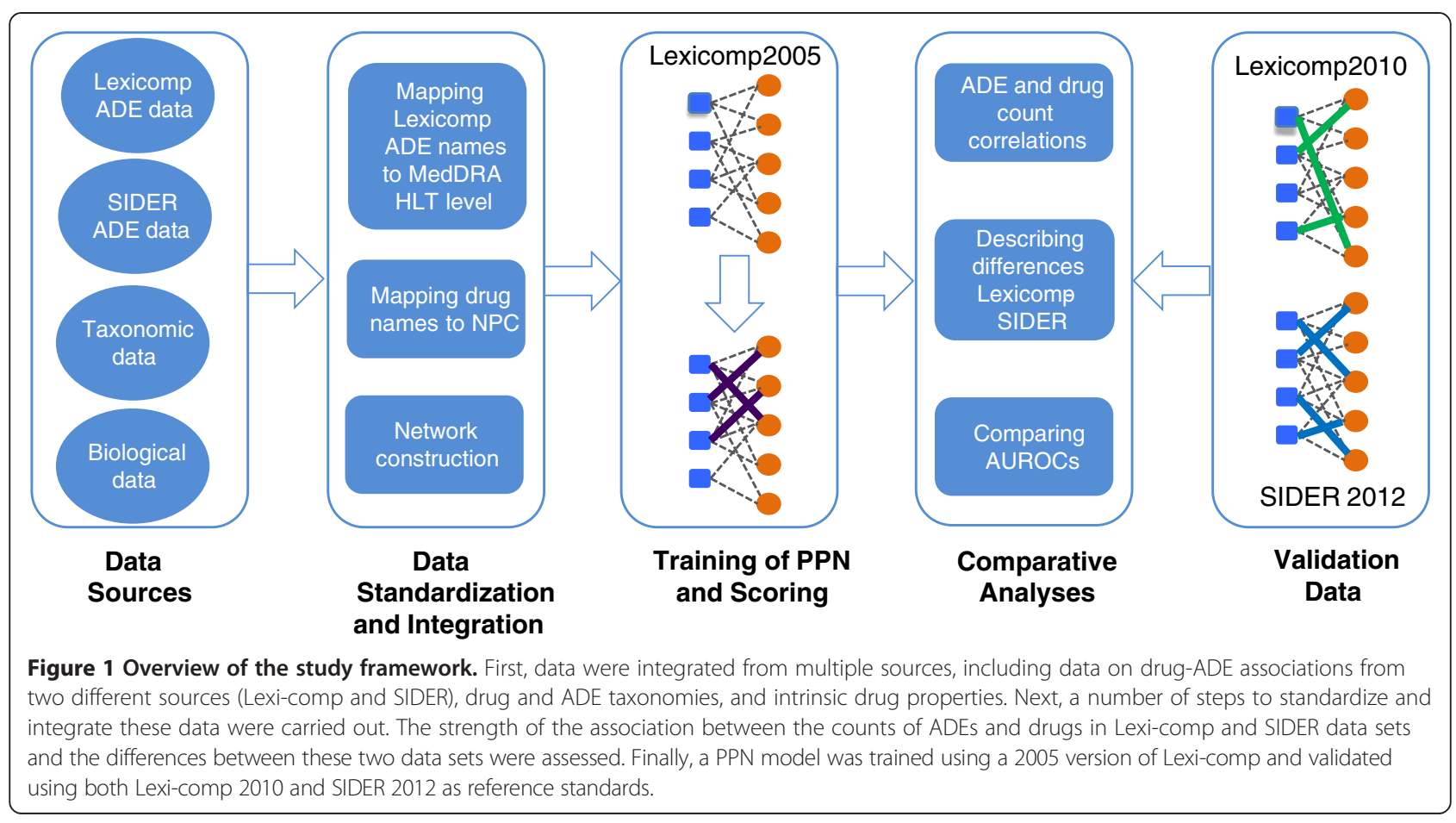


ADEs (per drug) and drugs (per ADE) in Lexi-comp and SIDER data sets. We also identified the difference between the two data sets and characterized it in terms of drug categories, ADE categories and ADE frequencies. Finally, we trained a PPN model using a 2005 version of Lexi-comp data and validated the prediction performance of PPN using both Lexi-comp 2010 and SIDER 2012 as reference standards.

\section{Data description}

The NCGC Pharmaceutical Collection (NPC) resource [31] was used to identify different forms of drug names that refer to a common "active pharmaceutical ingredient”. The Medical Dictionary for Regulatory Activities (MedDRA) standard (www.meddra.org) was used to code ADE names. Drug attributes were extracted from World Health Organization Anatomical Therapeutic Chemical Classification System (ATC) (www.whocc.no/atc), University of Alberta DrugBank (www.drugbank.ca), and National Center for Biotechnology Information's Pubchem Compound (www.ncbi.nlm.nih.gov/pccompound).

The Lexi-comp ADE data were extracted from LexiDrugs $^{\oplus}$ (http://www.lexi.com), a commercial database widely used in hospitals today. For this study, we had access to data on 809 Lexi-comp drugs. For each drug, we were provided with two text fields extracted, respectively, from 2005 and 2010 versions of Lexi-Drugs ${ }^{\oplus}$. Each of these text fields integrates ADE information from drug package inserts, relevant clinical trials, case studies and post-marketing reports. We extracted the ADE names contained in each field and mapped them to MedDRA Preferred Term (PT) level. In order to compress the space of all possible drug-ADE pairs, each PT was further mapped to one or more High-Level Terms (HLT) in the MedDRA hierarchy [21]. As an example, the PT "myocardial infarction" was mapped to the following two HLTs: "ischaemic coronary artery disorders", and "coronary necrosis and vascular insufficiency". Since several different PTs could typically map to the same HLT, this mapping compresses the space of possible drug-ADE pairs, thereby reducing the computational time needed to train and validate the prediction model. The main trade-off of such mapping is that more complex follow-up investigations would be needed to evaluate a predicted drug-HLT pair because only a subset of the PTs mapping to the predicted HLT may actually be associated with the drug. After these pre-processing steps, the final Lexi-comp data used in our study consisted of two lists of pairs of the form (drug name, HLT name) - one list corresponding to 2005 and another to 2010.

The SIDER data used here is the most recent version of the publicly available SIDER2 database, released in October 2012. We downloaded these data from the
FTP site ftp://sideeffects.embl.de/SIDER/2012-10-17/. Although earlier versions of SIDER going back to 2009 are available, SIDER2 is the first version of the database providing MedDRA-coded ADEs, making it suitable for this comparative study. SIDER integrates ADE information contained in package inserts and post-marketing reports using five main public sources: British Columbia Cancer Agency (www.bccancer.bc.ca), Facts@FDA (http://www.fda.gov), FDA Center for Drug Evaluation and Research (http://www.fda.gov), FDA MedWatch (http://www.fda.gov/) [32].

To enable the comparison of Lexi-comp and SIDER data, we standardized the drug names in both data sets using the NPC resource [31], and mapped SIDER ADE names from the Preferred Term level to the High-Level Terms level of MedDRA. The final pre-processed SIDER data used in our study consisted of a lists of pairs of the form (drug name, HLT name) corresponding to year 2012.

\section{Overview of PPNs}

Predictive Pharmacosafety Networks (PPN) [21] are predictive models that exploit the overall network structure of all known drug-ADE relationships and combine it with inherent attributes of drugs and adverse events in order to predict unknown adverse events. Rather than waiting for sufficient post-marketing evidence to accumulate for a specific ADE, this predictive approach relies on leveraging contextual information from previously known drug-safety relationships, and thus has the potential to predict certain candidate ADEs earlier than they can be detected by existing pharmacovigilance methods.

Here, we provide a brief overview of the PPN model; complete details of the model, including a full specification of the data sets used to train the model, are given in Cami et al. [21]. Using logistic regression, we model the presence or absence of drug-ADE associations $Y_{i j}, i=1$, ...,number of drugs, $j=1, \ldots$, number of ADEs, as a Bernoulli random variable and a function of three types of covariates. Network covariates depend only on the structure of the bipartite drug-ADE network. Taxonomic covariates depend on the structure of the drug-ADE network and on ATC and MedDRA codes. Intrinsic covariates depend on the structure of the drug-ADE network and on the intrinsic drug properties. Model fitting is carried out by maximum likelihood. After the model is estimated, each drug-ADE pair $(i, j)$ not reported to be an association in the training data is scored using the predicted probabilities generated by the model.

Cami et al. [21] used the Lexi-comp 2005 ADE data to form a bi-partite network that contained 39,591 links among 809 drugs and 852 HLTS. The drug and ADE attributes described above were integrated with the nodes of this network. Twelve predictor variables were then 
computed and a logistic regression (LR) model was estimated. This estimated LR model achieved an area under the Receiver Operating Characteristic curve (AUROC) of 0.87 in predicting the 10,845 drug-ADE associations that were newly reported in the 2010 version of Lexi-comp.

As case studies, eight prominent drug-ADE associations discovered during the period 2006-2010 were identified by two pharmacologists. For each case study, the specificity and the positive predictive value corresponding to the score generated by PPN were computed. It was found that the specificities corresponding to the model-generated scores were consistently high, providing additional support on the utility of the model. For example, the pair (norfloxacin, tendon ruptures) achieved a specificity of 0.95 , while (zonisamide, suicidal ideation) a specificity of 0.93 .

\section{Comparative analysis}

Since the Lexi-comp data available to us consisted of two snapshots from 2005 and 2010, ideally the comparison of Lexi-comp and SIDER would have been based on two SIDER snapshots from 2005 and 2010. However, the MedDRA-coded SIDER data available to us consisted of only one snapshot from 2012. Due to this restriction, we designed the comparative analysis as follows. We first identified the Lexi-comp 2010 drugs and ADEs from Cami et al. [21] study that were also included in SIDER 2012. Our goal was to assess the concordance of the sets of associations newly reported between these common drugs and ADEs in Lexi-comp 2010 and SIDER 2012, as well as the impact of data set choice on the prediction performance of PPN. In this analysis, we did not address any discordance between the sets of drug-ADE associations formed by drugs or ADEs that were included in only one of the two data sets.

In the first part of the comparative analysis, we assessed the strength of the association between ADE (or, drug) counts in Lexi-comp 2010 and SIDER 2012 by computing Spearman rank correlation. Next, we computed the difference between the Lexi-comp 2010 and SIDER 2012 data sets and characterized it in terms of drug categories, ADE categories and ADE frequencies. As described earlier, both Lexi-comp and SIDER use package inserts as the primary source of information. However, we expected that these data could differ for a number of reasons. First, SIDER 2012 may include new drug-ADE associations that were discovered after 2010 and thus could not be reported in the Lexi-comp data sets. Second, both Lexi-comp and SIDER supplement the package-inserts with other information extracted from various sources, as described earlier. Third, the mapping of ADE names to MedDRA- which, was independently implemented in the two data sets - is a non- deterministic process. There are numerous ADE names that appear in the original data sources for whom there is no exact match to MedDRA and for whom the most appropriate MedDRA code is determined either algorithmically or based on expert opinion.

In the second part of the comparative analysis, we trained a PPN model using Lexi-comp 2005 and then assessed the prediction performance of the model using Lexi-comp 2010 and SIDER 2012, respectively, as validation sets.

In the Discussion section, we explain how the data restriction mentioned earlier, i.e. the availability of only one SIDER snapshot taken at a different time point from either Lexi-comp snapshot, impacts the interpretation of our results.

\section{Results}

\section{Common drugs and ADEs}

Out of 809 Lexi-comp drugs used in the study by Cami et al. [21], 695 (86\%) were also included in SIDER; out of 852 HLTs used in [21], 765 (90\%) were also included in SIDER. In the reminder of this section, we compare the drug-ADE associations reported in Lexi-comp 2010 and SIDER 2012 between these common 695 drugs and 765 HLTs.

\section{Correlation between drug and ADE counts}

The Spearman correlation between the ADE counts in the Lexi-comp 2010 and SIDER 2012 data sets was 0.84 (95\% CI: 0.82-0.86) (Figure 2(A)), while the Spearman correlation between the drug counts in these two data sets was 0.92 (95\% CI: 0.91-0.93) (Figure 2(B)). For comparison, we also computed the Spearman correlations of $\mathrm{ADE}$ and drug counts between Lexi-comp 2005 and Lexi-comp 2010 data sets. For these two data sets, the correlations were, respectively, 0.87 and 0.99 .

\section{Differences between Lexi-comp and SIDER data sets}

Figure 2 indicates that drug and ADE counts generated from SIDER 2012 are generally higher than the corresponding counts from Lexi-comp 2010. In aggregate, we found that relative to the Lexi-comp 2005 data snapshot, Lexi-comp 2010 and SIDER 2012 introduced new drugADE associations at a mean rate of 1,973 and 4,810 per year, respectively. To better understand this difference between Lexi-comp 2010 and SIDER 2012, we computed the percentage of SIDER-only associations in each ATC top-level category, each MedDRA top-level category, and each ADE frequency group ("postmarketing", "frequent", "rare", "potential", or exact number).

We found that the drug categories with the highest percentages of SIDER-only ADEs were drugs targeting 

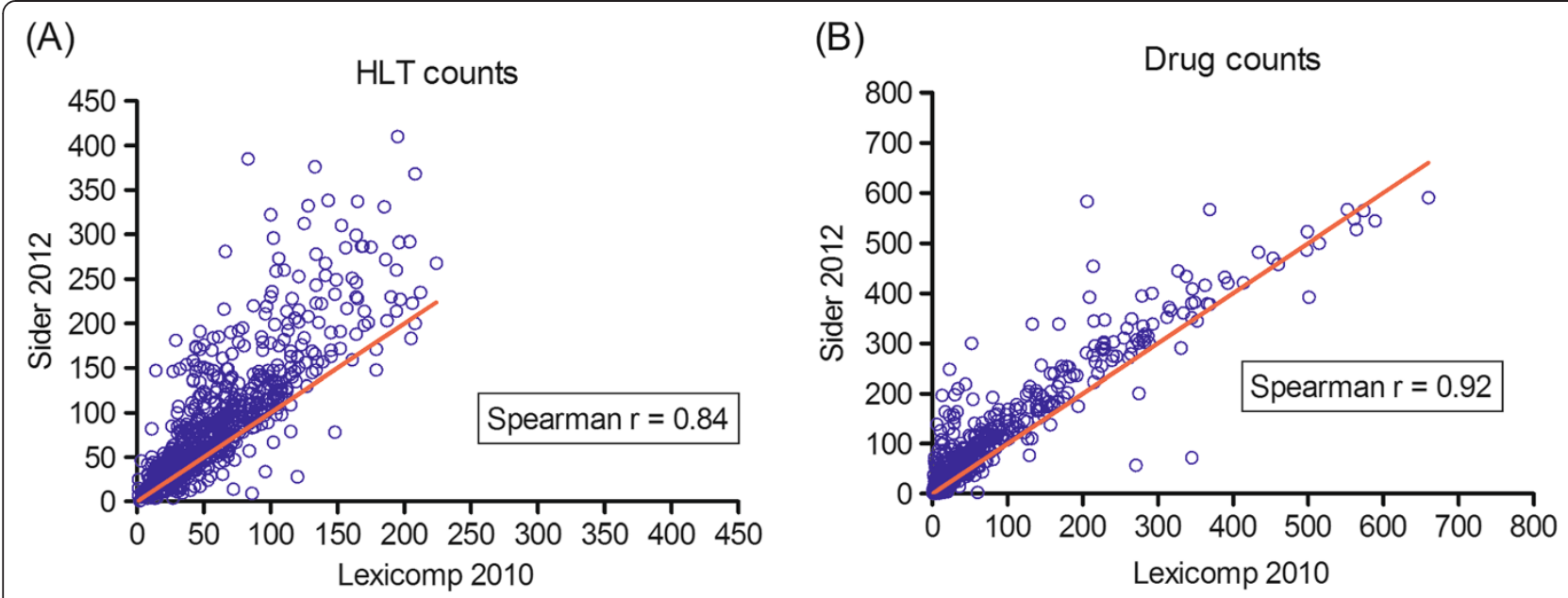

Figure 2 Scatter plots of HLT and drug counts from Lexi-comp 2010 and SIDER 2012. (A) HLT counts and (B) drug counts.

the Nervous System and Antiinfective drugs (21.9\% and $11.4 \%$, respectively, Table 1), while the ADE categories with the highest percentages of SIDER-only drugs were Nervous System Disorders and Gastrointestinal Disorders $(8.9 \%$ and $8.7 \%$, respectively, Table 2$)$. With regards to ADE frequency classes, we found that for $63 \%$ of SIDER-only associations the frequency of ADE was missing. Of the remaining SIDER-only associations, the type having the highest percentage was "postmarketing" (46\%), followed by "infrequent" (16\%), "exact number" (16\%), "rare" (11\%), "potential" (8\%), and "frequent" (3\%).

\section{Table 1 Number and percentage of SIDER-only} associations by ATC top-level category

\begin{tabular}{ll}
\hline ATC top-level category & $\begin{array}{l}\text { Number (percent) of } \\
\text { SIDER-only associations }\end{array}$ \\
\hline Nervous system & $7641(21.9 \%)$ \\
Antiinfectives for systemic use & $3993(11.4 \%)$ \\
Cardiovascular system & $3894(11.2 \%)$ \\
Antineoplastic and immunomodulating & $3704(10.6 \%)$ \\
agents & \\
Sensory organs & $3672(10.5 \%)$ \\
Alimentary tract and metabolism & $2573(7.4 \%)$ \\
Musculo-skeletal system & $2300(6.6 \%)$ \\
Genito-urinary system and sex hormones & $2157(6.2 \%)$ \\
Dermatologicals & $2024(5.8 \%)$ \\
Respiratory system & $1378(3.9 \%)$ \\
Systemic hormonal preparations (excl sex & $693(2.0 \%)$ \\
hormones) & \\
Blood and blood forming organs & $514(1.5 \%)$ \\
Various & $219(0.6 \%)$ \\
Antiparasitic products insecticides and & $146(0.4 \%)$ \\
repellents & \\
\hline
\end{tabular}

\section{Prediction performance of PPN}

We compared the predictive scores generated by the PPN model trained on data from Lexi-comp 2005 with the newly introduced drug-ADE associations in Lexicomp 2010 and SIDER 2012 data sets. We found a very minor overall change in the Area Under the Receiver Operating Characteristic curve (AUROC) of the PPN model when SIDER 2012 data were used for validation instead of Lexi-comp 2010 data: 0.84 vs. 0.85, respectively (Figure 3). Stratification of AUROC by top-level MedDRA category - i.e. System Organ Class (SOC) showed that there is no clear relationship between the number of SIDER-only associations in a category and the change in AUROC when SIDER is used for validation instead of Lexi-comp (Table 3). In fact, the relative change in AUROC was less than 5\% for all but six SOCs which are rather general and not specifically related to a body organ or system: 1) Congenital, familial and genetic disorders (relative change 34\%), 2) Surgical and medical procedures (relative change 20\%), 3) General disorders (relative change 12\%), 4) Injury, poisoning and procedural disorders (relative change 7\%), 5) Investigations (relative change 7\%),6) Pregnancy, puerperium and perinatal conditions (relative change 5.1\%).

\section{Discussion}

This study aimed to systematically assess the concordance between Lexi-comp and SIDER ADE data sets, as well as the impact of using each data set in the prediction performance of PPN model. Our main result was that ADE and drug counts in the Lexi-comp 2010 and SIDER 2012 data sets were highly correlated and that the AUROC of the PPN model changed very little (approximately 1.1\%) when SIDER 2012 was used for validation instead of Lexi-comp 2010. 


\begin{tabular}{ll} 
Table 2 Number and percentage of SIDER-only \\
associations by MedDRA top-level category \\
\hline MedDRA top-level category & $\begin{array}{l}\text { Number (percent) of } \\
\text { SIDER-only associations }\end{array}$ \\
\hline Nervous system disorders & $3091(8.9 \%)$ \\
Gastrointestinal disorders & $3038(8.7 \%)$ \\
Skin and subcutaneous tissue disorders & $2948(8.4 \%)$ \\
General disorders & $2487(7.1 \%)$ \\
Respiratory, thoracic and mediastinal & $2309(6.6 \%)$ \\
disorders & \\
Vascular disorders & $2251(6.4 \%)$ \\
Psychiatric disorders & $2069(5.9 \%)$ \\
Musculoskeletal and connective tissue & $1764(5.1 \%)$ \\
disorders & $1751(5.0 \%)$ \\
Eye disorders & $1713(4.9 \%)$ \\
Infections and infestations & $1477(4.2 \%)$ \\
Investigations & $1417(4.1 \%)$ \\
Metabolism and nutrition disorders & $1287(3.7 \%)$ \\
Blood and lymphatic system disorders & $1211(3.5 \%)$ \\
Cardiac disorders & $1116(3.2 \%)$ \\
Renal and urinary disorders & $1014(2.9 \%)$ \\
Reproductive system and breast disorders & $784(2.2 \%)$ \\
Immune system disorders & $627(1.8 \%)$ \\
Neoplasms benign, malignant and & $614(1.8 \%)$ \\
unspecified & $524(1.5 \%)$ \\
Hepatobiliary disorders & $409(1.2 \%)$ \\
Injury, poisoning and procedural disorders & $388(1.1 \%)$ \\
Endocrine disorders & \\
Ear and labyrinth disorders & \\
Surgical and medical procedures & \\
\hline & \\
\hline &
\end{tabular}

While we found overall concordance, there were also differences between the Lexi-comp 2010 and SIDER 2012 data sets. These differences were most pronounced for Nervous System and Anti-infective drugs, for Gastrointestinal and Nervous System ADEs, and for "postmarketing" ADEs. Our results suggest that the differences between the two data sets do not simply arise from the two-year time lag between them. Indeed, the correlations of drug and ADE counts were higher between the two Lexi-comp snapshots (separated by five years) than they were between Lexi-comp 2010 and SIDER 2012. Further, relative to Lexi-comp 2005, SIDER 2012 introduces new associations at a higher rate than Lexi-comp 2010. As discussed earlier, other factors that could have introduced these differences include the use of various

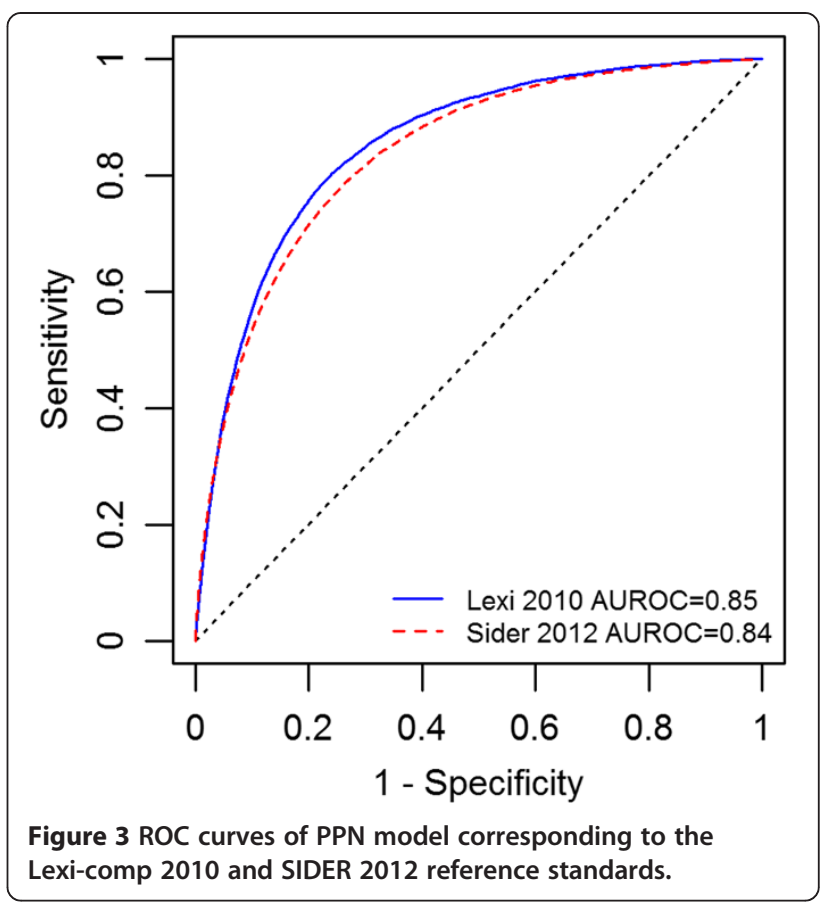

sources to supplement package-insert information and the independent mapping of ADE names to MEdDRA.

The observations of high overall concordance between Lexi-comp and SIDER, and high robustness of the PPN model under two different validation sets, are not affected by the time lag between the two data sets. In fact, the presence of the time lag makes the concordance and robustness conclusions even stronger than they would be if the same results were obtained by comparing data from the same year. On the other hand, the differences between the two data sets should be interpreted with caution as it is not clear to what extent they are accounted for by the time lag and to what extent by other factors. The interpretation of these differences is also hindered by the high proportion of missing ADE frequency data $(63 \%)$.

While this manuscript was under preparation, Lin et al. [27] published a new study in which they developed an "external link prediction" method for unknown drug-ADE associations. Using two snapshots of data based on the intersection of SIDER with FAERS 2005 and FAERS 2011, respectively, they carried out a simulated prospective validation of a subset of PPN covariates analogous to the validation by Cami et al. [21]. The training set in the study by Lin et al. consisted of 422 drugs and 462 ADEs. These authors found that in that data set, the chosen subset of PPN covariates achieved an AUROC of 0.75, while the "external link prediction" method achieved an AUROC of 0.83 . Thus, the study by Lin et al. using a different ADE data source, different validation year and different drug and $\mathrm{ADE}$ sets provides 
Table 3 AUROC of PPN by MedDRA top-level category

\begin{tabular}{|c|c|c|}
\hline \multirow[t]{2}{*}{ MedDRA top-level category } & \multirow{2}{*}{$\begin{array}{l}\text { Lexi-comp } 2010 \\
\text { AUROC }(95 \% \mathrm{Cl})\end{array}$} & \multirow{2}{*}{$\begin{array}{l}\text { SIDER } 2012 \\
\text { AUROC }(95 \% \mathrm{Cl})\end{array}$} \\
\hline & & \\
\hline Nervous system disorders & $0.85(0.84-0.86)$ & $0.84(0.84-0.85)$ \\
\hline Gastrointestinal disorders & $0.85(0.84-0.86)$ & $0.84(0.83-0.85)$ \\
\hline Skin and subcutaneous tissue disorders & $0.85(0.84-0.86)$ & $0.85(0.85-0.86)$ \\
\hline General disorders & $0.86(0.84-0.87)$ & $0.76(0.75-0.77)$ \\
\hline Respiratory, thoracic and mediastinal disorders & $0.80(0.79-0.82)$ & $0.78(0.77-0.79)$ \\
\hline Vascular disorders & $0.84(0.83-0.86)$ & $0.83(0.82-0.84)$ \\
\hline Psychiatric disorders & $0.83(0.82-0.85)$ & $0.81(0.81-0.82)$ \\
\hline Musculoskeletal and connective tissue disorders & $0.82(0.80-0.84)$ & $0.83(0.82-0.84)$ \\
\hline Eye disorders & $0.79(0.77-0.81)$ & $0.83(0.82-0.84)$ \\
\hline Infections and infestations & $0.85(0.83-0.86)$ & $0.85(0.84-0.86)$ \\
\hline Investigations & $0.86(0.84-0.88)$ & $0.80(0.79-0.81)$ \\
\hline Metabolism and nutrition disorders & $0.81(0.79-0.83)$ & $0.82(0.81-0.83)$ \\
\hline Blood and lymphatic system disorders & $0.87(0.86-0.89)$ & $0.88(0.87-0.88)$ \\
\hline Cardiac disorders & $0.83(0.81-0.85)$ & $0.84(0.82-0.85)$ \\
\hline Renal and urinary disorders & $0.86(0.84-0.87)$ & $0.85(0.83-0.86)$ \\
\hline Reproductive system and breast disorders & $0.85(0.82-0.87)$ & $0.86(0.84-0.87)$ \\
\hline Immune system disorders & $0.88(0.87-0.90)$ & $0.90(0.89-0.91)$ \\
\hline Neoplasms benign, malignant and unspecified & $0.80(0.74-0.85)$ & $0.78(0.76-0.80)$ \\
\hline Hepatobiliary disorders & $0.85(0.83-0.88)$ & $0.83(0.81-0.84)$ \\
\hline Injury, poisoning and procedural disorders & $0.86(0.84-0.89)$ & $0.80(0.78-0.82)$ \\
\hline Endocrine disorders & $0.82(0.79-0.85)$ & $0.85(0.83-0.86)$ \\
\hline Ear and labyrinth disorders & $0.86(0.83-0.89)$ & $0.84(0.82-0.86)$ \\
\hline Surgical and medical procedures & $0.82(0.73-0.89)$ & $0.66(0.60-0.72)$ \\
\hline Pregnancy, puerperium and perinatal conditions & $0.78(0.63-0.93)$ & $0.74(0.66-0.82)$ \\
\hline Congenital, familial and genetic disorders & $0.91(0.83-0.98)$ & $0.60(0.53-0.66)$ \\
\hline
\end{tabular}

Note: Rows are shown in decreasing order of the number of SIDER-only associations. Bold-face: Six MedDRA categories with the highest AUROC difference between SIDER 2012 and Lexi-comp 2010.

an independent confirmation of the robustness of PPN variables with respect to the choice of ADE data set.

Recently, Tatonetti et al. [24] published a method to extract potentially significant drug-ADE associations from FAERS and a new accompanying data set of such associations (OFFSIDES). Similarly, Cheng et al. [25] developed a new drug-ADE data set named MetaADEDB by integrating information from SIDER, CTD (ctdbase. org), and OFFSIDES, and utilizing Medical Subject Headings $(\mathrm{MeSH})$ to annotate compounds and diseases. We believe that these data integration, standardization and annotation efforts are important steps toward the development of improved reference standards for drugADE associations.

\section{Conclusions}

In summary, we have conducted a study that systematically compared two drug safety data sets and assessed the impact of data set choice on the prediction performance of the PPN predictive model. Overall, we found a high concordance between the two data sets and only a minor impact on the prediction performance of PPN. However, we also identified a number of key differences between the two data sets. We believe it is important for researchers, drug safety professionals and public health officials to be aware of such differences, especially in modeling applications aimed at specific drug and ADE categories, and a wide range of studies aimed at ADE prediction models.

\section{Competing interests}

The authors declare that they have no competing interests.

Authors' contributions

$A C$ and BR acquired the data, developed the proposed methods and designed the study. AC implemented the methods and performed the experiments. AC and BR wrote the manuscript. Both authors read and approved the final manuscript. 


\section{Acknowledgements}

This work was supported by the National Library of Medicine (grant number: 4R00LM011014) and the National Institute of General Medical Sciences (grant numbers: 1R01GM085421, R01GM89731).

Received: 2 May 2014 Accepted: 18 August 2014

Published: 22 August 2014

\section{References}

1. Johnson JA, Bootman JL: Drug-related morbidity and mortality: a cost-ofillness model. Arch Intern Med 1995, 155(18):1949.

2. Lazarou J, Pomeranz BH, Corey PN: Incidence of adverse drug reactions in hospitalized patients. JAMA 1998, 279(15):1200-1205.

3. Chiatti C, Bustacchini S, Furneri G, Mantovani L, Cristiani M, Misuraca C, Lattanzio F: The economic burden of inappropriate drug prescribing, lack of adherence and compliance, adverse drug events in older people. Drug Saf 2012, 35(1):73-87.

4. Gyllensten $\mathrm{H}$, Jonsson AK, Rehnberg C, Carlsten A: How are the costs of drug-related morbidity measured?: a systematic literature review. Drug Saf 2012, 35(3):207-219.

5. Wu C, Bell CM, Wodchis WP: Incidence and economic burden of adverse drug reactions among elderly patients in Ontario Emergency Departments. Drug Saf 2012, 35(9):769-781.

6. Dearden JC: In silico prediction of drug toxicity. J Comput Aided Mol Des 2003, 17(2):119-127.

7. Hamon J, Whitebread S, Techer-Etienne V, Le Coq H, Azzaoui K, Urban L: In vitro safety pharmacology profiling: what else beyond hERG? Future Med Chem 2009, 1(4):645-665.

8. Fieldent MR, Kolaja KL: The role of early in vivo toxicity testing in drug discovery toxicology. Expert Opin Drug Saf 2008, 7(2):107-110.

9. Brown JS, Kulldorff M, Chan KA, Davis RL, Graham D, Pettus PT, Andrade SE, Raebel MA, Herrinton L, Roblin D, Boudreau D, Smith D, Gurwitz JH, Gunter MJ, Platt R: Early detection of adverse drug events within populationbased health networks: application of sequential testing methods. Pharmacoepidemiol Drug Saf 2007, 16(12):1275-1284.

10. Noren GN, Edwards IR: Modern methods of pharmacovigilance: detecting adverse effects of drugs. Clin Med 2009, 9(5):486-489.

11. Frantz S: Pharma's year of trouble and strife. Nat Rev Drug Discov 2006, 5(1):7-9.

12. Hughes B: 2007: Spotlight on drug safety. Nat Rev Drug Discov 2008, 7(1):5-7.

13. Fung M, Thornton A, Mybeck K, Wu JH-h, Hornbuckle K, Muniz E: Evaluation of the characteristics of safety withdrawal of prescription drugs from worldwide pharmaceutical markets-1960 to $1999^{*}$. Drug Inf J 2001, 35(1):293-317.

14. MacDonald JS, Robertson RT: Toxicity testing in the 21st century: a view from the pharmaceutical industry. Toxicol Sci 2009, 110(1):40-46.

15. Kola I, Landis J: Can the pharmaceutical industry reduce attrition rates? Nat Rev Drug Discov 2004, 3(8):711-716.

16. Paul SM, Mytelka DS, Dunwiddie CT, Persinger CC, Munos BH, Lindborg SR, Schacht AL: How to improve R\&D productivity: the pharmaceutical industry's grand challenge. Nat Rev Drug Discov 2010, 9(3):203-214.

17. Bai JP, Abernethy DR: Systems pharmacology to predict drug toxicity: integration across levels of biological organization*. Annu Rev Pharmacol Toxicol 2013, 53:451-473.

18. Hopkins AL: Network pharmacology: the next paradigm in drug discovery. Nat Chem Biol 2008, 4(11):682-690.

19. Azuaje F: Drug interaction networks: an introduction to translational and clinical applications. Cardiovasc Res 2013, 97(4):631-641.

20. Atias N, Sharan R: An algorithmic framework for predicting side effects of drugs. J Comput Biol 2011, 18(3):207-218.

21. Cami A, Arnold A, Manzi S, Reis B: Predicting adverse drug events using pharmacological network models. Sci Transl Med 2011, 3(114):114ra127.

22. Huang $L-C$, Wu $X$, Chen JY: Predicting adverse side effects of drugs. BMC Genomics 2011, 12(Suppl 5):S11.

23. Liu M, Wu Y, Chen Y, Sun J, Zhao Z, Chen X-w, Matheny ME, Xu H: Large-scale prediction of adverse drug reactions using chemical, biological, and phenotypic properties of drugs. J Am Med Inform Assoc 2012, 19(e1):e28-e35.

24. Tatonetti NP, Patrick PY, Daneshjou R, Altman RB: Data-driven prediction of drug effects and interactions. Sci Trans/ Med 2012, 4(125):125ra131.
25. Cheng F, Li W, Wang X, Zhou Y, Wu Z, Shen J, Tang Y: Adverse drug events: database construction and in silico prediction. J Chem Inf Model 2013, 53(4):744-752

26. Duran-Frigola M, Aloy P: Analysis of chemical and biological features yields mechanistic insights into drug side effects. Chem Biol 2013, 20(4):594-603.

27. Lin J, Kuang Q, Li Y, Zhang Y, Sun J, Ding Z, Li M: Prediction of adverse drug reactions by a network based external link prediction method. Anal Methods 2013, 5(21):6120-6127.

28. Shimazawa $\mathrm{R}$, Ikeda M: Safety information in drug labeling: a comparison of the USA, the UK, and Japan. Pharmacoepidemiol Drug Saf 2013, 22(3):306-318.

29. Wang LM, Wong M, Lightwood JM, Cheng CM: Black box warning contraindicated comedications: concordance among three major drug interaction screening programs. Ann Pharmacother 2010, 44(1):28-34

30. Duke J, Friedlin J, Li X: Consistency in the safety labeling of bioequivalent medications. Pharmacoepidemiol Drug Saf 2013, 22(3):294-301.

31. Huang R, Southall N, Wang Y, Yasgar A, Shinn P, Jadhav A, Nguyen D-T, Austin CP: The NCGC pharmaceutical collection: a comprehensive resource of clinically approved drugs enabling repurposing and chemical genomics. Sci Transl Med 2011, 3(80):80ps16.

32. Kuhn M, Campillos M, Letunic I, Jensen LJ, Bork P: A side effect resource to capture phenotypic effects of drugs. Mol Syst Biol 2010, 6:343.

doi:10.1186/1472-6947-14-74

Cite this article as: Cami and Reis: Concordance and predictive value of two adverse drug event data sets. BMC Medical Informatics and Decision Making 2014 14:74.

\section{Submit your next manuscript to BioMed Central and take full advantage of:}

- Convenient online submission

- Thorough peer review

- No space constraints or color figure charges

- Immediate publication on acceptance

- Inclusion in PubMed, CAS, Scopus and Google Scholar

- Research which is freely available for redistribution 